\title{
Cyclooxygenase mediates cardioprotection of angiotensin-(1-7) against ischemia/reperfusion-induced injury through the inhibition of oxidative stress
}

\author{
XINXUE LIAO ${ }^{1,2 *}$, LICHUN WANG ${ }^{1,2^{*}}$, CHUNTAO YANG $^{3}$, JIANGUI HE $^{1,2}$, XIUYU WANG $^{3}$, RUIXIAN GUO $^{3}$, \\ AIPING LAN ${ }^{3}$, XIAOBIAN DONG ${ }^{1,2}$, ZHANLI YANG $^{3}$, HUAQIAO WANG $^{4}$, JIANQIANG FENG ${ }^{3}$ and HONG MA MA $^{1,2}$ \\ Departments of ${ }^{1}$ Cardiovasology, and ${ }^{2}$ Hypertension and Vascular Diseases, The First Affiliated Hospital; \\ Departments of ${ }^{3}$ Physiology and ${ }^{4}$ Anatomy, Zhongshan School of Medicine, Sun Yat-Sen University, \\ Guangzhou, Guangdong 510080, P.R. China
}

Received May 9, 2011; Accepted August 17, 2011

DOI: $10.3892 / \mathrm{mmr} .2011 .570$

\begin{abstract}
Angiotensin (Ang)-(1-7) exhibits cardioprotective effects in myocardial ischemia reperfusion (I/R)-induced injury. However, the roles of oxidation and cyclooxygenase (COX) in the cardioprotection of Ang-(1-7) remain unclear. This study was conducted to investigate whether oxidation and COX were involved in the cardioprotection of Ang-(1-7) against I/Rinduced injury in isolated rat hearts. The hearts were subjected to $15 \mathrm{~min}$ regional ischemia followed by $30 \mathrm{~min}$ reperfusion. Myocardial I/R treatment induced significant cardiac dysfunction, including ventricular arrhythmia (VA) and a reduction of left ventricular systolic pressure (LVSP), cardiomyocyte apoptosis and oxidative stress, manifesting as an increase in malondialdehyde (MDA) production and a decrease in superoxide dismutase (SOD) activity. Pretreatment of the hearts with $1.0 \mathrm{nmol} / \mathrm{l} \mathrm{Ang-(1-7)} \mathrm{for} 30 \mathrm{~min}$ prior to ischemia considerably attenuated I/R-induced VA, apoptosis and MDA production, and enhanced LVSP and SOD activity. These cardioprotective effects of Ang-(1-7) were antagonized by the intraperitoneal injection of $5 \mathrm{mg} / \mathrm{kg}$ body weight indomethacin (IDM, a COX inhibitor), presenting as an enhancement of VA, apoptosis and MDA production as well as a reduction of LVSP and SOD activity. In conclusion, COX mediated Ang-(1-7)-induced cardioprotection via its antioxidative mechanism.
\end{abstract}

Correspondence to: Professor Hong Ma, Departments of Cardiovasology, Hypertension and Vascular Diseases, The First Affiliated Hospital of Sun Yat-sen University, Guangzhou, Guangdong 510080, P.R. China

E-mail: sums-mahong@21cn.com

Professor Jianqiang Feng, Department of Physiology, Zhongshan Medical College, Sun Yat-sen University, Guangzhou, Guangdong 510080, P.R. China

E-mail: fengjq-sums@163.com

*Contributed equally

Key words: angiotensin-(1-7), apoptosis, cyclooxygenase, ischemia/ reperfusion, oxidative stress, prostaglandin

\section{Introduction}

Reperfusion of ischemic myocardium further aggravates tissue damage induced by ischemia despite providing cells with oxygen and trophic substances. This damage is partly due to the migration of neutrophils from the vasculature to the tissue and the production of reactive oxygen species (ROS) $(1,2)$. Superoxide anion is a type of major ROS in ischemia-reperfusion $(\mathrm{I} / / \mathrm{R})$ of vascular tissue that originates from the catalysis of NADPH oxidase in the neutrophil (2) or leakage of the electron transport chain in the mitochondria (3). Since the heart is particularly susceptible to oxidative damage, I/R-triggered oxidative stress induces cellular apoptosis in the myocardium, which may be attenuated by free radical scavengers (4). Apoptosis may be responsible for 5-33\% of cardiomyocyte loss in animal models in response to I/R (5). A portion of myocardial death upon $\mathrm{I} / \mathrm{R}$ has been found to be apoptotic, contributing to the development of cardiac dysfunction and progression of cardiac failure. In addition, various studies indicated that the rennin-angiotensin system (RAS) is activated during myocardial ischemia $(6,7)$ and that angiotensin II (AngII) plays a significant role in myocardial I/R-induced injury (8). The inhibition of RAS (9) and AngII receptors $(8,10)$ was shown to be beneficial in protecting against myocardial injury induced by $\mathrm{I} / \mathrm{R}$, indicating that the compounds with opposing effects of AngII may be protective for myocardial I/R-induced injury.

Angiotensin-(1-7) [Ang-(1-7)] is one of the bioactive components of RAS that can be formed from AngI and AngII (11). In addition, Ang-(1-7) exhibits vasodilatory and antiproliferative activities in contrast to the constrictive and proliferative effects of AngII. In human forearm resistant vessels, Ang-(1-7) has been shown to antagonize vasoconstriction by AngII (12). One study also indicated that Ang-(1-7) inhibited the trophic actions of AngII and reduced the expression of the mitogenic effects of normal serum and platelet-derived growth factor in the culture vascular smooth muscle cells (VSMCs), revealing the antiproliferative actions of Ang-(1-7) (13). Previously, a number of studies reported that the heart is a major target for the actions of Ang-(1-7), where Ang-(1-7) has beneficial 
effects on the coronary bed and cardiac function $(14,15)$. Ang-(1-7) not only reduced the incidence and duration of cardiac arrhythmia (16), but also improved the post-ischemic contractile function in isolated perfused rat hearts (17). These effects were completely blocked by the Ang-(1-7) receptor Mas antagonist A-779 (16,17). However, whether or not antioxidation is involved in the cardioprotection of Ang-(1-7) against myocardial injury induced by $\mathrm{I} / \mathrm{R}$ remains to be fully elucidated.

Cyclooxygenase (COX) exists in two isoforms: a constitutive $\mathrm{COX}(\mathrm{COX}-1)$ and an inducible $\mathrm{COX}(\mathrm{COX}-2)$, which are responsible for the production of prostaglandin (PG). Although studies have indicated that the overexpression of $\mathrm{COX} / \mathrm{PG}$ mediated inflammatory insult in a number of tissue types, including CA1 hippocampal neurons (18) and skin keratinocytes (19), a number of studies of the cardiovascular system indicated that overexpression of $\mathrm{COX} / \mathrm{PG}$ provided marked protection $(20,21)$.

The aim of this study was to investigate whether Ang-(1-7) protected the hearts from I/R-induced myocardial injury including cardiomyocyte apoptosis and, if so, whether oxidative stress and COX were involved in the cardioprotective effects of Ang-(1-7).

\section{Materials and methods}

Animals. Forty-three male Sprague Dawley rats (8 weeks of age) weighing 280-340 g, were used in the present study. The animal experiments were conducted in accordance with guidelines for the human use of laboratory animals of Sun Yat-Sen University and approved by local authorities.

Isolated rat heart preparation. The rats were anesthetized by an intraperitoneal injection of $10 \%$ chloral hydrate. To anticoagulate, 500 units of heparin were administered intraperitoneally to the rats. Thoracic surgery was performed to remove the heart. The hearts from heparinized rats were rapidly excised and immediately mounted on Langendorff's apparatus. The hearts were perfused at a constant pressure of $65 \mathrm{mmHg}$ with $\mathrm{KH}$ buffer $\left(95 \% \mathrm{O}_{2}, 5 \% \mathrm{CO}_{2} 37^{\circ} \mathrm{C}\right)$. Two thin silver electrodes were fixed at the ventricular apex and origin/aorta to record the electrocardiogram (ECG) and heart rate. A plastic catheter with a latex balloon was inserted into the left ventricle to measure developed left ventricular systolic pressure (LVSP).

Experimental groups. The rats were randomly distributed in 5 groups, and each group consisted of 8-12 animals. The rats were intraperitoneally injected with $5 \mathrm{mg} / \mathrm{kg}$ body weight indomethacin (IDM) (Sigma-Aldrich Co., St. Louis, MO, USA) to inhibit COX or normal saline (NS) at an equal volume. Following anesthetization, the hearts were excised from the chests and subjected to I/R or non-I/R treatment in the presence or absence of pretreatment with $1.0 \mathrm{nmol} / \mathrm{l} \mathrm{Ang-(1-7)}$ (Sigma-Aldrich Co.) in KH buffer. To produce ischemia and reperfusion in isolated hearts, a 6-0 silk suture was placed around the left anterior descending coronary artery to form a snare, and the preparation was allowed to stabilize for $30 \mathrm{~min}$. Ischemia was induced by tightening the threads of the coronary suture and was maintained for $15 \mathrm{~min}$. Reperfusion was obtained by cutting the ligature around the coronary artery and lasted for $30 \mathrm{~min}$. The hearts were grouped as follows: control group: 8 hearts treated with non-I/R following injection of NS. Vehicle group: 9 hearts treated with I/R following injection of NS. Ang-(1-7) group: 10 hearts treated with I/R in the presence of pretreatment with Ang-(1-7) for $30 \mathrm{~min}$ before regional ischemia following the injection of NS. IDM + Ang-(1-7) group: 8 hearts treated in the same manner as hearts in the Ang-(1-7) group following injection of IDM. IDM group: 8 hearts treated as the vehicle group following injection of IDM. Injections were administered intraperitoneally.

Analysis of ventricular arrhythmia. ECG was recorded using two thin silver electrodes fixed at the ventricular apex and origin/aorta. Ventricular arrhythmia (VA) triggered by I/R, including ventricular tachycardia (VT), defined as three or more consecutive premature ventricular contractions, and ventricular fibrillation (VF), defined as an irregular modulating baseline, were evaluated using ECG recording for the duration of reperfusion. The degree of VA was scored from 0-12: no VT or VF was scored as 0; VA lasting $<3$ min was scored as 2; VA lasting for 3-6, 7-10, 11-15, 16-20, 21-25 and >25 min was scored as 4, 6, 8, 10, 11 and 12 , respectively.

Measurement of LVSP. During the experiments, LVSP was measured by the BL-420F system (Chengdu Technology and Market Co., Ltd., Chengdu, China) at three indicated time points: $10 \mathrm{~min}$ prior to ischemia, $15 \mathrm{~min}$ after ischemia and $30 \mathrm{~min}$ after the reperfusion.

Determination of oxidative stress. Malondialdehyde (MDA) content and superoxide dismutase (SOD) activity were used as indices to assess oxidative stress. Following the indicated treatments, the left ventricular anterior wall was collected and homogenated. Measurement was performed according to the manufacturer's instructions (Jiancheng Bioengineering Institute, Nanjing, China) using a microplate reader (Molecular Devices, Sunnyvale, CA, USA).

Detection of apoptosis by TUNEL staining. Following the indicated treatments, the left ventricular anterior wall was dissected from the hearts, and cut into 5- $\mu \mathrm{m}$ sections to detect apoptosis. The tissue sections were stained using terminal deoxynucleotidyl transferase-mediated dUTP nick end-labeling (TUNEL) staining (Sigma-Aldrich Co.). The number of TUNEL-positive cardiac myocyte nuclei was counted in at least 20 high-power fields (HPF) for each heart under a light microscope at a magnification of $x 400$. The TUNEL-positive cardiomyocyte nuclei from $20 \mathrm{HPF}$ per rat were averaged.

Statistical analysis. Data are expressed as the mean \pm standard deviation (SD). The significance of inter-group differences was evaluated using one-way analysis of variance and Scheffe's test for post hoc comparisons. $\mathrm{P}<0.05$ was considered to be statistically significant.

\section{Results}

COX was involved in antiarrhythmias of Ang-(1-7) in the $I / R$-injured hearts. I/R-induced VA was observed during reperfusion for $30 \mathrm{~min}$. As shown in Fig. 1, when the hearts were subjected to I/R treatment, the VA score was markedly 


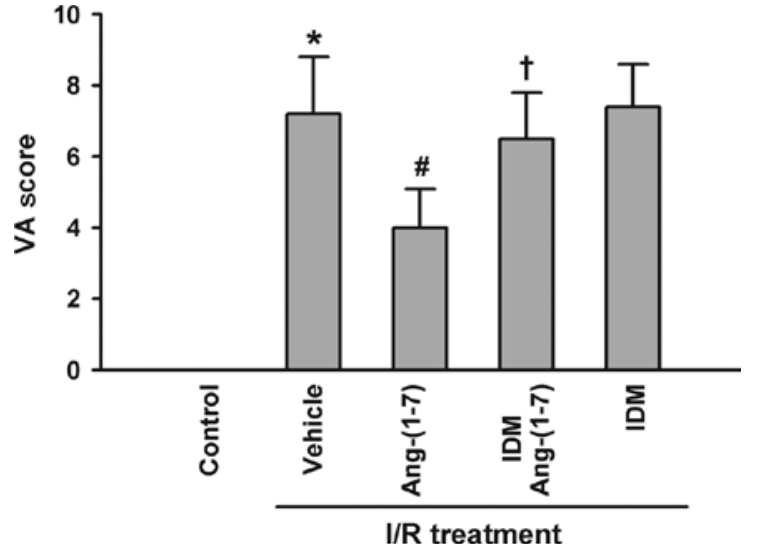

Figure 1. Role of cyclooxygenase (COX) in angiotensin (Ang)-(1-7)-induced improvement of ventricular arrhythmias in ischemia reperfusion (I/R)stimulated hearts. The rats were injected intraperitoneally with $5 \mathrm{mg} / \mathrm{kg}$ body weight indomethacin (IDM) or NS and the hearts were then removed from the chests and subjected to $\mathrm{I} / \mathrm{R}$ or non-I/R treatment in the presence or absence of pretreatment with $1.0 \mathrm{nmol} / 1$ Ang-(1-7) in $\mathrm{KH}$ buffer. Control group $(\mathrm{n}=8)$ hearts treated with non-I/R following NS injection. Vehicle group $(n=9)$ : hearts treated with I/R following NS injection. Ang-(1-7) group ( $\mathrm{n}=10)$ : hearts treated with $\mathrm{I} / \mathrm{R}$ in the presence of Ang-(1-7) pretreatment following NS injection. IDM + Ang-(1-7) group $(n=8)$ : hearts treated with $I / R$ in the presence of Ang-(1-7) pretreatment following IDM injection. IDM group $(n=8)$ : hearts treated with I/R following IDM injection. Ventricular arrhythmia (VA) score was recorded in the hearts. Injections were administered intraperitoneally. ${ }^{\text {}} \mathrm{P}<0.01$ compared with the control group; ${ }^{\#} \mathrm{P}<0.01$ compared with the vehicle group; ${ }^{\top} \mathrm{P}<0.05$ compared with the Ang-(1-7) group.

increased. Prior to exposure to I/R, the hearts were treated with Ang-(1-7) at a concentration of $1.0 \mathrm{nmol} / \mathrm{l}$ in $\mathrm{KH}$ buffer. The increased VA score was markedly attenuated by pretreatment with Ang-(1-7). Notably, this effect was antagonized by the intraperitoneal administration of $5 \mathrm{mg} / \mathrm{kg}$ body weight IDM (a COX inhibitor) to the rats, which did not affect the VA score alone. The result indicates that Ang-(1-7) exerts an antiarrhythmic effect at least partly by activating COX.

COX-mediated Ang-(1-7)-induced increase in LVSP. As shown in Table I, no significant difference was observed in the LVSP of hearts between the indicated groups prior to I/R treatment. When the hearts were subjected to ischemia for $15 \mathrm{~min}$, LVSP was markedly reduced. Similarly, 20 min of reperfusion followed by ischemia also significantly reduced LVSP. The decreased LVSP induced by ischemia or reperfusion was inhibited by pretreatment of hearts with Ang-(1-7) at a concentration of $1.0 \mathrm{nmol} / 1$ in $\mathrm{KH}$ buffer. Notably, inhibition of COX by administration of $5 \mathrm{mg} / \mathrm{kg}$ body weight IDM to the rats statistically attenuated the Ang-(1-7)-triggered improvement of LVSP in I/R-damaged hearts, which alone did not affect an I/R-induced decrease in LVSP when administered alone. These results indicate that Ang-(1-7) protects heart against the decrease in LVSP induced by ischemia and reperfusion, and COX only mediates the cardioprotection of Ang-(1-7) against reperfusion-induced decrease in LVSP.

COX was involved in Ang-(1-7)-induced inhibition of apoptosis in the I/R-injured hearts. To assess whether I/R-induced heart dysfunction was associated with cardiomyocyte apoptosis, the TUNEL assay was performed. Markedly more
A

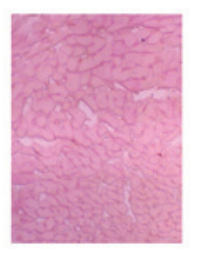

$\mathbf{F}$
B

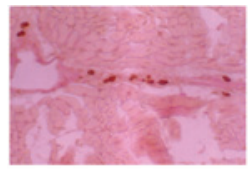

C

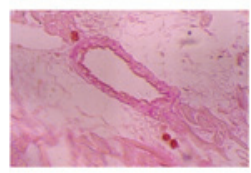

D

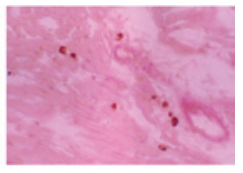

$\mathbf{E}$

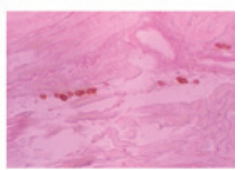

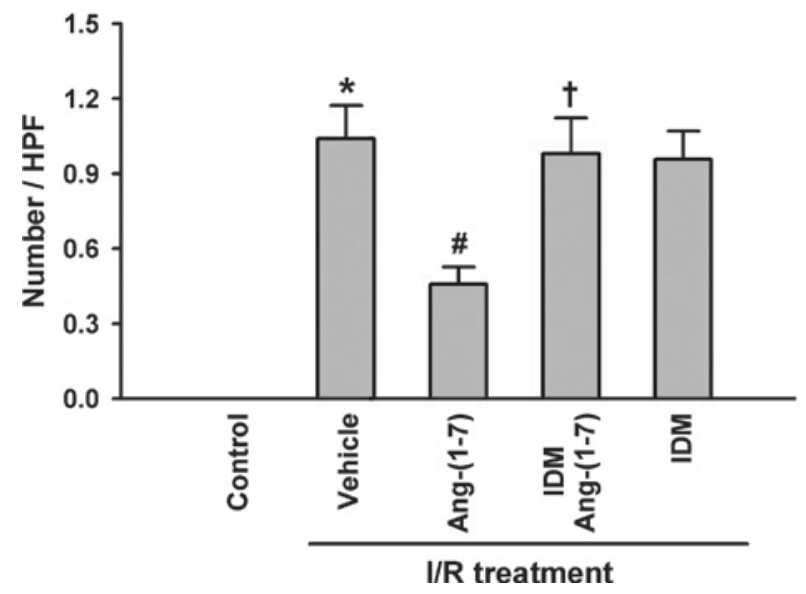

Figure 2. Roles of cyclooxygenase (COX) in angiotensin (Ang)-(1-7)-induced antiapoptosis in ischemia reperfusion (I/R)-insulted hearts. Rats were injected intraperitoneally with $5 \mathrm{mg} / \mathrm{kg}$ body weight indomethacin (IDM) or NS, and then the hearts were removed from the chests and subjected to $\mathrm{I} / \mathrm{R}$ or non-I/R treatment in the presence or absence of pretreatment with $1.0 \mathrm{nmol} / 1 \mathrm{Ang}-(1-7)$ in $\mathrm{KH}$ buffer. (A) Control group ( $\mathrm{n}=8$ ): hearts treated with non-I/R following NS injection. (B) Vehicle group $(n=9)$ : hearts treated with I/R following NS injection. (C) Ang-(1-7) group ( $\mathrm{n}=10)$ : hearts treated with I/R in the presence of Ang-(1-7) pretreatment following NS injection. (D) IDM + Ang-(1-7) group (n=8): hearts treated with $I / R$ in the presence of Ang-(1-7) pretreatment following IDM injection. (E) IDM group $(n=8)$ : hearts treated with I/R following IDM injection. (A-E) Representative images of TUNEL assay to show cardiomyocyte apoptosis. (F) Number of TUNELpositive cardiomyocyte nuclei (apoptotic cells) was counted in at least 20 HPF for each heart, and averaged. Injections were administered intraperitoneally. ${ }^{~} \mathrm{P}<0.01$ compared with the control group, ${ }^{\prime} \mathrm{P}<0.01$ compared with the vehicle group and ${ }^{\dagger} \mathrm{P}<0.01$ compared with the Ang-(1-7) group.

TUNEL-positive cardiomyocytes were observed in the I/R treatment group (Fig. 2B and F) compared to the control group (Fig. 2A). The number of TUNEL-positive cardiomyocytes induced by $\mathrm{I} / \mathrm{R}$ was significantly reduced by pretreatment with Ang-(1-7) at $1.0 \mathrm{nmol} / \mathrm{l}$ in $\mathrm{KH}$ buffer (Fig. 2C and F). Injection (intraperitoneal) of IDM, a COX inhibitor, at $5 \mathrm{mg} / \mathrm{kg}$ body weight, which alone did not alter the I/R-induced cardiomyocyte apoptosis (Fig. 2D), statistically attenuated the antiapoptotic effect of Ang-(1-7) (Fig. 2E and F).

COX was involved in the antioxidation of Ang-(1-7) in I/Rinsulted hearts. MDA is an end-product of the peroxidation of cell membrane lipids induced by ROS and is thought to be one of the biochemical markers of I/R-induced myocardial oxidative injury. Fig. 3A shows that treatment of heart with I/R significantly enhanced MDA production in myocardial tissue, which was significantly inhibited by pretreatment with Ang-(1-7) at $1.0 \mathrm{nmol} / \mathrm{l}$ in $\mathrm{KH}$ buffer. Inhibition of COX by 
A

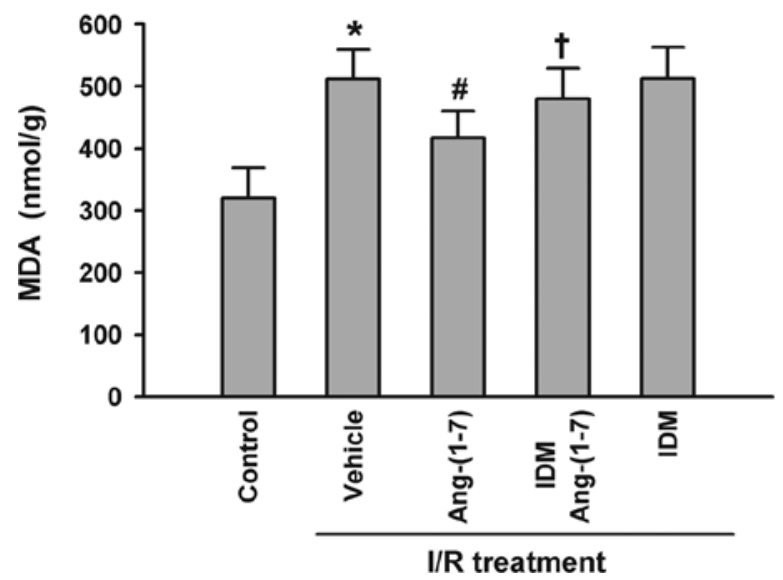

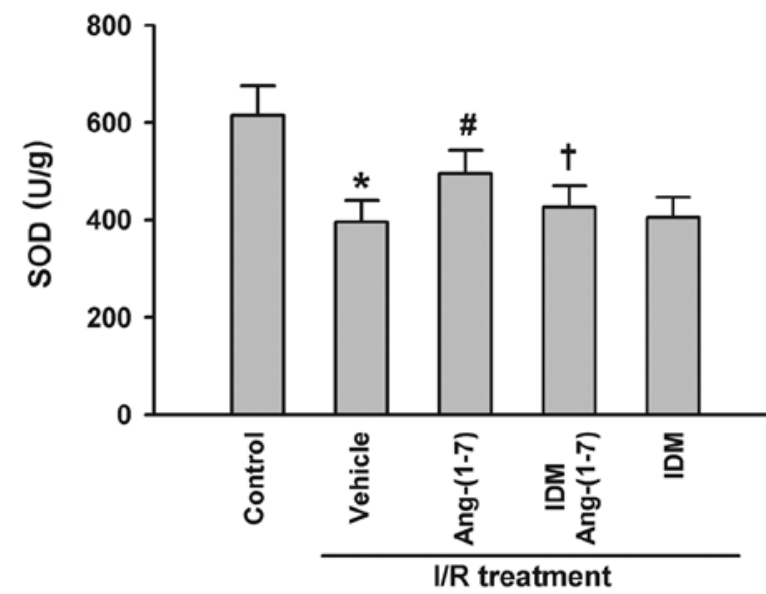

Figure 3. Roles of cyclooxygenase (COX) in angiotensin (Ang)-(1-7)-induced antioxidation in ischemia reperfusion (I/R)-insulted hearts. Rats were injected with $5 \mathrm{mg} / \mathrm{kg}$ body weight indomethacin (IDM) or NS intraperitoneally. The hearts were then removed from the chests and subjected to I/R or non-I/R treatment in the presence or absence of pretreatment with $1.0 \mathrm{nmol} / 1$ Ang-(1-7) in $\mathrm{KH}$ buffer. Control group (n=8): hearts treated with non-I/R following NS injection. Vehicle group ( $\mathrm{n}=9$ ): hearts treated with $\mathrm{I} / \mathrm{R}$ following NS injection. Ang-(1-7) group (n=10): hearts treated with I/R in the presence of Ang-(1-7) pretreatment following NS injection. IDM + Ang-(1-7) group ( $\mathrm{n=8)}$ : hearts treated with I/R in the presence of Ang-(1-7) pretreatment following IDM injection. IDM group ( $\mathrm{n}=8$ ): hearts treated with I/R following IDM injection. (A) Malondialdehyde (MDA) level and (B) superoxide dismutase (SOD) activity were detected. Injections were administered intraperitoneally. ${ }^{*} \mathrm{P}<0.01$ compared with the control group; ${ }^{\#} \mathrm{P}<0.05$ compared with the vehicle group; and ${ }^{\dagger} \mathrm{P}<0.05$ compared with the Ang-(1-7) group.

Table I. Role of COX in Ang-(1-7)-induced improvement of LVSP in the I/R-stimulated hearts ( $\mathrm{mmHg}$ ).

\begin{tabular}{lrccc}
\hline Group & N & Pre-I/R & $\begin{array}{c}\text { Ischemia } \\
(15 \mathrm{~min})\end{array}$ & $\begin{array}{c}\text { Reperfusion } \\
(30 \mathrm{~min})\end{array}$ \\
\hline Vehicle & 9 & $73.8 \pm 7.8$ & $52.8 \pm 5.1^{\mathrm{b}}$ & $46.7 \pm 4.6^{\mathrm{b}}$ \\
Ang-(1-7) & 10 & $75.0 \pm 6.7$ & $57.0 \pm 4.8^{\mathrm{a}, \mathrm{c}}$ & $59.0 \pm 5.3^{\mathrm{a}, \mathrm{d}}$ \\
Ang-(1-7) & & & & \\
+ IDM & 8 & $70.6 \pm 7.2$ & $53.4 \pm 5.8^{\mathrm{b}}$ & $47.6 \pm 6.1^{\mathrm{b}, \mathrm{e}}$ \\
IDM & 9 & $73.1 \pm 7.0$ & $51.9 \pm 4.6^{\mathrm{b}}$ & $45.9 \pm 5.1^{\mathrm{b}}$ \\
\hline
\end{tabular}

COX,cyclooxygenase; Ang, angiotensin;LVSP,left ventricularsystolic pressure; I/R, ischemia reperfusion; IDM, indomethacin. Rats were intraperitoneally injected with $5 \mathrm{mg} / \mathrm{kg}$ body weight IDM or NS. The hearts were then removed from the chests and subjected to ischemia and/or reperfusion treatment in the presence or absence of pretreatment with $1.0 \mathrm{nmol} / 1 \mathrm{Ang}-(1-7)$ in $\mathrm{KH}$ buffer. During various periods, LVSP of isolated hearts was measured. ${ }^{\mathrm{P}} \mathrm{P}<0.05$, ${ }^{\mathrm{b}} \mathrm{P}<0.01$, compared with the Pre-I/R group, ${ }^{\mathrm{C}} \mathrm{P}<0.05,{ }^{\mathrm{d}} \mathrm{P}<0.01$ compared with the vehicle group and ${ }^{\mathrm{e}} \mathrm{P}<0.05$ compared with the Ang-(1-7) group.

intraperitoneal injection of IDM at $5 \mathrm{mg} / \mathrm{kg}$ body weight, which alone did not affect the I/R-induced MDA overproduction, partly abrogated Ang-(1-7)-induced inhibition of MDA overproduction in I/R-insulted myocardial tissue. This result indicates that Ang-(1-7) possesses antioxidation at least partly by activating COX pathway.

SOD is a significant antioxidant system. As shown in Fig. 3B, following exposure to I/R, SOD activity in myocardial tissue was obviously decreased compared with that of the control group. Notably, I/R-induced decrease in SOD activity was significantly attenuated by pretreatment with Ang-(1-7) at $1.0 \mathrm{nmol} / 1$ in $\mathrm{KH}$ buffer. Similarly, the Ang-(1-7)-triggered improvement of SOD activity was partly abolished by the inhibition of COX with an intraperitoneal injection of IDM at $5 \mathrm{mg} / \mathrm{kg}$ body weight, which alone did not alter the decreased SOD activity induced by I/R treatment.

\section{Discussion}

The present study provides direct evidence that Ang-(1-7), a novel vasodilating peptide derived from AngI and AngII (11), protects isolated rat hearts against I/R-induced injury. It attenuated I/R-induced VA and cardiomyocyte apoptosis as well as MDA production and enhanced LVSP and SOD activity. These beneficial effects may be mediated by activating the COX/PG pathway.

$\mathrm{I} / \mathrm{R}$ insult is one of the most significant etiological factors of heart dysfunction. In the present study, significant myocardial dysfunction, including VA and reduction of LVSP, was predominantly observed at reperfusion of the ischemic myocardium. This is in agreement with numerous reports, indicating reperfusion as a key trigger of a number of events leading to myocardial dysfunction associated with I/R injury (22). ROS was reportedly produced mostly at reperfusion of the ischemic organ rather than during ischemia (23).

Previously, it was thought that an oxygen supply below a critical value led to cardiomyocyte death by necrosis. However, findings of various studies have shown that apoptosis, another death mode, occurred in adult mammalian hearts during I/R and cardiac overload (24). In the current study, we also found that $\mathrm{I} / \mathrm{R}$ treatment induced significant apoptosis, as determined by TUNEL assay. Our findings are comparable with those of the previous studies mentioned above $(24,25)$.

Various studies have indicated that AngII plays a role in myocardial ischemia $(6,7)$ and $\mathrm{I} / \mathrm{R}$-induced damage (8). Angiotensin-converting enzyme inhibitor (ACEI) and AngII type 2 receptor antagonist were effective in reducing the I/R-induced injury (8). Since Ang-(1-7) opposed the physiological effects of AngII, including its vasoconstrictive, 
proliferative and tropic effects $(12,13)$, we hypothesized that Ang-(1-7) protects cardiomyocytes against I/R-induced injury. Results of this study show that Ang-(1-7) was afforded cardioprotective effects against myocardial I/R-induced damage, including attenuation of VA and cardiomyocyte apoptosis, as well as enhancement of LVSP. The present study provides significant evidence to support the finding that angiotensinconverting enzyme 2 , which acts as a carboxypeptidase clearing a single residue from AngII to generate Ang-(1-7) (26), is an essential regulator of heart function (27). Our data also increase understanding of the physiological functions of Ang-(1-7), particularly in the cardiovascular system. Findings of previous studies have shown that Ang-(1-7) had an antiarrhythmic effect $(16,26)$ and improved the post-ischemic contractile function (17) in isolated perfused rat hearts. In addition, Ang-(1-7) receptor Mas played a significant role in the maintenance of post-ischemic cardiac function (28). These reports were in concordance with the findings of the present study.

To investigate the mechanism underlying the cardioprotection of Ang-(1-7), an experiment was performed to examine whether: i) I/R induced changes in MDA production and SOD activity; ii) Ang-(1-7) affected the changes in MDA level and SOD activity induced by I/R; and iii) the COX/PG pathway was involved in the cardioprotective effects of Ang-(1-7). Our data revealed that $\mathrm{I} / \mathrm{R}$ induced an increase in MDA production and a decrease in SOD activity. MDA is an end-product of the peroxidation of cell membrane lipids caused by ROS. SOD is one of the most significant antioxidant systems, functioning as a ROS scavenger. Our data therefore indicated that I/R-induced overproduction of ROS, such as superoxide anion, may be enhanced when SOD activity was overwhelmed. The imbalance between oxidation and antioxidation, SOD, resulted in superoxide-mediated injury. Numerous studies have shown that the excessive release of superoxide anion contributed to the I/R-induced heart injury (29). Our results are in agreement with previous studies regarding the protection of a selective SOD mimetic (30). Significantly, we observed that Ang-(1-7) protected against myocardial I/R-induced injury, accompanied by the attenuation of MDA production and enhancement of SOD activiy, indicating that one of the mechanisms of the cardioprotection of Ang-(1-7) was associated with its antioxidant effects. Mounting evidence has indicated that a variety of antioxidants and antioxidative enzymes play a significant role in myocardial protection against acute stress such as ischemia or hypoxia (31). SOD is believed to be a key antioxidative enzyme against free radical attack during the $\mathrm{I} / \mathrm{R}$ period. SOD catalyzes the dismutation reaction (32). In heart, several studies have shown that dismutation of superoxide with the native SOD $(33,34)$ or with Cu-Zn SOD $(35)$ protected against myocardial I/R-induced injury. SOD and catalase were also shown to reduce I/R-induced apoptotic cell death and MDA production (4). M40403, a selective SOD mimetic, was reported to significantly attenuate the extent of myocardial damage, mast cell degranulation and the incidence of VA and to improve survival (30). Based on these findings, it was presumed that the enhancement of SOD activity may be an important mechanism associated with the cardioprotective effects of Ang-(1-7).

Notably, we observed that pretreatment with IDM, a COX inhibitor, significantly attenuated the cardioprotective effects of Ang-(1-7), including enhancement of VA, cardiomyocyte apoptosis, and MDA level and reduction of LVSP and SOD activity, indicating that PGs may be involved in the cardioprotective effects of Ang-(1-7). COX, the rate-limiting enzyme in PG synthesis, has been shown to play a role in preventing apoptosis (36). COX exists in two isoforms: a constitutive COX-1 and an inducible COX-2. It was demonstrated that COX-1- or COX-2-knockout mice were susceptible to cardiac I/R-induced injury (37). Inhibition of COX-2 enhanced cell death, particularly apoptotic cell death (38). PGs also protected cells from injury in a number of tissues, including the cardiomyocyte. One study suggested that Ang-(1-7) stimulated prostaglandin $E_{2}$ release from rat VSMCs (39). The vasodilation of pial arteries by Ang-(1-7) and the depressor component of the response to Ang-(1-7) were suppressed by pretreatment with the COX inhibitor IDM, indicating that these hemodynamic responses to Ang-(1-7) were mediated by PGs $(40,41)$. Furthermore, it was indicated that IDM antagonized not only the antiarrhythmic effect of Ang-(1-7) (16), but also the beneficial effects of Ang-(1-7) on systolic function during ischemia and subsequent reperfusion in isolated rat hearts (17). We therefore believe that Ang-(1-7)-induced cardioprotective and antioxidant effects were mediated at least partly by the activation of COX/PG.

In conclusion, the present study clearly indicated that Ang-(1-7) protected against myocardial I/R-induced injury by attenuation of VA and MDA production as well as cardiomyocyte apoptosis and enhancement of LVSP and SOD activity. The cardioprotective and antioxidant effects of Ang-(1-7) may be mediated by COX/PG. Knowledge of cardioprotection and mechanisms of Ang-(1-7) may offer a novel clue for the treatment of ischemic heart disease.

\section{Acknowledgements}

This study was supported by the Guangdong Science and Technology Planning Project Fund (nos. 2010B080701105, 2009B080701014 and 2007B080701030).

\section{References}

1. Bolli R: Oxygen-derived free radicals and myocardial reperfusion injury: an overview. Cardiovasc Drugs Ther 5 (Suppl 2): 249-268, 1991.

2. Marzocca C, Vannacci A, Cuzzocrea S, et al: Effects of the SOD mimetic, M40403, on prostaglandin production in an in vivo model of ischemia and reperfusion in rat heart. Inflamm Res 52 (Suppl 1): 23-24, 2003.

3. Boveris A: Mitochondrial production of superoxide radical and hydrogen peroxide. Adv Exp Med Biol 78: 67-82, 1977.

4. Galang N, Sasaki H and Maulik N: Apoptotic cell death during ischemia/reperfusion and its attenuation by antioxidant therapy. Toxicology 148: 111-118, 2000.

5. Bialik S, Geenen DL, Sasson IE, et al: Myocyte apoptosis during acute myocardial infarction in the mouse localizes to hypoxic regions but occurs independently of p53. J Clin Invest 100: 1363-1372, 1997.

6. Reiss K, Capasso JM, Huang HE, et al: ANG II receptors, c-myc, and c-jun in myocytes after myocardial infarction and ventricular failure. Am J Physiol 264: H760-H769, 1993.

7. Noda K, Sasaguri M, Ideishi M, et al: Role of locally formed angiotensin II and bradykinin in the reduction of myocardial infarct size in dogs. Cardiovasc Res 27: 334-340, 1993.

8. Wang LX, Ideishi M, Yahiro E, et al: Mechanism of the cardioprotective effect of inhibition of the renin-angiotensin system on ischemia/reperfusion-induced myocardial injury. Hypertens Res 24: 179-187, 2001. 
9. Liu KL, Sassard J and Benzoni D: In the Lyon hypertensive rat renal function alterations are angiotensin II dependent. Am J Physiol 271: R346-R351, 1996.

10. Yahiro E, Ideishi M, Wang LX, et al: Reperfusion-induced arrhythmias are suppressed by inhibition of the angiotensin II type 1 receptor. Cardiology 99: 61-67, 2003.

11. Chappell MC, Tallant EA, Brosnihan KB, et al: Processing of angiotensin peptides by NG108-15 neuroblastoma $\mathrm{x}$ glioma hybrid cell line. Peptides 11: 375-380, 1990.

12. Ueda S, Masumori-Maemoto S, Ashino K, et al: Angiotensin-(1-7) attenuates vasoconstriction evoked by angiotensin II but not by noradrenaline in man. Hypertension 35: 998-1001, 2000.

13. Tallant EA, Diz DI and Ferrario CM: State-of-the-Art lecture. Antiproliferative actions of angiotensin-(1-7) in vascular smooth muscle. Hypertension 34: 950-957, 1999.

14. Ferrario CM, Chappell MC, Tallant EA, et al: Counterregulatory actions of angiotensin-(1-7). Hypertension 30: 535-541, 1997.

15. Santos RA, Ferreira AJ, Pinheiro SV, et al: Angiotensin-(1-7) and its receptor as a potential targets for new cardiovascular drugs. Expert Opin Investig Drugs 14: 1019-1031, 2005.

16. Ferreira AJ, Santos RA and Almeida AP: Angiotensin-(1-7): cardioprotective effect in myocardial ischemia/reperfusion. Hypertension 38: 665-668, 2001.

17. Ferreira AJ, Santos RA and Almeida AP: Angiotensin-(1-7) improves the post-ischemic function in isolated perfused rat hearts. Braz J Med Biol Res 35: 1083-1090, 2002.

18. Nakayama M, Uchimura K, Zhu RL, et al: Cyclooxygenase-2 inhibition prevents delayed death of CA1 hippocampal neurons following global ischemia. Proc Natl Acad Sci USA 95: 10954-10959, 1998.

19. Yang CT, Ling HZ and Zhang MF: Oxidative stress mediates chemical hypoxia-induced injury and inflammation by activating NF- $\mathrm{kb}-\mathrm{COX}-2$ pathway in HaCaT cells. Mol Cells 31: 531-538 2011.

20. Booth EA, Flint RR, Lucas KL, et al: Estrogen protects the heart from ischemia-reperfusion injury via COX-2-derived PGI2. J Cardiovasc Pharmacol 52: 228-235, 2008.

21. Kwak HJ, Park KM, Choi HE, et al: The cardioprotective effects of zileuton, a 5-lipoxygenase inhibitor, are mediated by COX-2 via activation of PKC delta. Cell Signal 22: 80-87, 2010.

22. Masini E, Pierpaoli S, Marzocca C, et al: Protective effects of a plant histaminase in myocardial ischaemia and reperfusion injury in vivo. Biochem Biophys Res Commun 309: 432-439, 2003.

23. Cuzzocrea S, Mazzon E, Dugo L, et al: Protective effects of a new stable, highly active SOD mimetic, M40401 in splanchnic artery occlusion and reperfusion. Br J Pharmacol 132: 19-29, 2001 .

24. Bromme HJ and Holtz J: Apoptosis in the heart: when and why? Mol Cell Biochem 163-164: 261-275, 1996.

25. Kajstura J, Cheng W, Reiss K, et al: Apoptotic and necrotic myocyte cell deaths are independent contributing variables of infarct size in rats. Lab Invest 74: 86-107, 1996.

26. Tipnis SR, Hooper NM, Hyde R, et al: A human homolog of angiotensin-converting enzyme. Cloning and functional expression as a captopril-insensitive carboxypeptidase. J Biol Chem 275: 33238-33243, 2000.
27. Trask AJ, Averill DB, Ganten D, et al: Primary role of angiotensinconverting enzyme-2 in cardiac production of angiotensin-(1-7) in transgenic Ren-2 hypertensive rats. Am J Physiol Heart Circ Physiol 292: H3019-H3024, 2007.

28. Castro CH, Santos RA, Ferreira AJ, et al: Effects of genetic deletion of angiotensin-(1-7) receptor Mas on cardiac function during ischemia/reperfusion in the isolated perfused mouse heart. Life Sci 80: 264-268, 2006.

29. Grill HP, Zweier JL, Kuppusamy P, et al: Direct measurement of myocardial free radical generation in an in vivo model: effects of postischemic reperfusion and treatment with human recombinant superoxide dismutase. J Am Coll Cardiol 20: 1604-1611, 1992.

30. Masini E, Cuzzocrea S, Mazzon E, et al: Protective effects of M40403, a selective superoxide dismutase mimetic, in myocardial ischaemia and reperfusion injury in vivo. Br J Pharmacol 136: 905-917, 2002.

31. Das DK, Prasad MR, Lu D, et al: Preconditioning of heart by repeated stunning. Adaptive modification of antioxidative defense system. Cell Mol Biol (Noisy-le-grand) 38: 739-749, 1992.

32. Greenlund LJ, Deckwerth TL and Johnson EM Jr: Superoxide dismutase delays neuronal apoptosis: a role for reactive oxygen species in programmed neuronal death. Neuron 14: 303-315, 1995.

33. Hangaishi M, Nakajima $\mathrm{H}$, Taguchi J, et al: Lecithinized $\mathrm{Cu}$, $\mathrm{Zn}$-superoxide dismutase limits the infarct size following ischemia-reperfusion injury in rat hearts in vivo. Biochem Biophys Res Commun 285: 1220-1225, 2001

34. Li Q, Bolli R, Qiu Y, et al: Gene therapy with extracellular superoxide dismutase protects conscious rabbits against myocardial infarction. Circulation 103: 1893-1898, 2001.

35. Mehta JL, Chen H, Li D, et al: Modulation of myocardial SOD and iNOS during ischemia-reperfusion by antisense directed at ACE mRNA. J Mol Cell Cardiol 32: 2259-2268, 2000.

36. Abbate A, Santini D, Biondi-Zoccai GG, et al: Cyclo-oxygenase-2 (COX-2) expression at the site of recent myocardial infarction: friend or foe? Heart 90: 440-443, 2004.

37. Camitta MG, Gabel SA, Chulada P, et al: Cyclooxygenase-1 and -2 knockout mice demonstrate increased cardiac ischemia/ reperfusion injury but are protected by acute preconditioning. Circulation 104: 2453-2458, 2001.

38. Adderley SR and Fitzgerald DJ: Oxidative damage of cardiomyocytes is limited by extracellular regulated kinases $1 / 2$-mediated induction of cyclooxygenase-2. J Biol Chem 274: 5038-5046, 1999.

39. Jaiswal N, Jaiswal RK, Tallant EA, et al: Alterations in prostaglandin production in spontaneously hypertensive rat smooth muscle cells. Hypertension 21: 900-905, 1993.

40. Benter IF, Diz DI and Ferrario CM: Cardiovascular actions of angiotensin(1-7). Peptides 14: 679-684, 1993.

41. Meng W and Busija DW: Comparative effects of angiotensin-(1-7) and angiotensin II on piglet pial arterioles. Stroke 24: 2041-2044, 1993. 